\title{
EVALUACIÓN DE ALGUNOS PARÁMETROS FÍSICOS DE Persea americana MEDIANTE UN EQUIPO DE MEDICIÓN DE POSTCOSECHA
}

\author{
EVALUATION OF SOME PHYSICAL PARAMETERS OF PERSEA \\ AMERICANA IN POST-HARVEST MEASUREMENT EQUIPMENT
}

Raul Cartagena Cutipa ${ }^{1}$ Percy Fermín Velázquez Ccosi ${ }^{2}$ Danny Daniel Carhuaz Valdez ${ }^{3}$ Hugo Javier Rivera Herrera ${ }^{4}$

Información del artículo: Recibido: 25/04/2020

Aceptado: 20/06/2020

\footnotetext{
1,3,4 Docente en la Facultad de Ingeniería, Universidad Privada de Tacna. E-mail:

${ }^{1}$ raulcart@hotmail.com, ${ }^{3}$ danielcarhuaz@hotmail, ${ }^{4}$ eshjrivera.upt@gmail.com ${ }^{2}$ Docente Ingeniería Agroindustrial, Universidad Nacional San Cristobal de Humanga. E-mail: percy.velasquez@unsch.edu.pe
} 


\section{Resumen}

Para de evaluar el comportamiento post cosecha de frutos climatéricos, se construyó un equipo experimental que determinase la intensidad de tasa respiratoria en partes por millón ( $\mathrm{ppm}$ ) de dióxido de carbono ( $\mathrm{CO} 2$ ) por unidad de masa y tiempo, para ello se utilizaron: un sensor $\mathrm{K}-33 \mathrm{ICB}$ para medición de $\mathrm{CO}$; bomba de aire marca Thomas 2000DV/0,5/E/LC; una trampa de agua de vidrio de $2,5 \mathrm{~cm}$ de diámetro y largo útil de $6 \mathrm{~cm}$; un filtro hidrofóbico de $0,22 \mu \mathrm{m}$; un filtro de partículas de $150 \mu \mathrm{m}$; una cámara de respiración con un volumen de 5,0 x10-3 m3 construida de vidrio con dos tabuladuras para intercambio gaseoso y conductos de látex. Para la adquisición de datos se empleó el software Gaslab ${ }^{\circledR}$. Con la finalidad de evaluar el equipo se seleccionaron frutos de palta Hass (Persea americana) en el grado de madurez fisiológica con 8,26 \% de aceite, posteriormente se acondicionó en la cámara de respiración con un peso inicial de un kilogramo, seguidamente se selló herméticamente para poner en funcionamiento el equipo para registrar la taza respiratoria durante 24 días, finalizando el periodo, el fruto entró en el proceso de climaterio y se determinó la madurez comercial con un contenido de aceite de 16,06 \%. El comportamiento de la tasa respiratoria de palta Hass fue típica de un fruto climatérico, presentando el pico climatérico en el día 20 con 244 x 103 ppm/ Kg. H. La pérdida fisiológica de peso y la firmeza, presentaron un comportamiento decreciente durante la etapa postcosecha de los frutos, mientras que la mayor concentración de aceite, se presentó a 24 días de postcosecha, etapa que coincidió con la madurez de consumo

Palabras claves: Palta Hass; Postcosecha; Tasa respiratoria.

\section{Abstract}

In order to evaluate the post-harvest behavior of climacteric fruits, an experimental team was constructed to determine the intensity of respiratory rate in parts per million (ppm) of carbon dioxide ( $\mathrm{CO} 2)$ per unit of mass and time, for which purpose they were used : a K33 ICB sensor for CO2 measurement; Thomas 2000DV / 0,5 / E / LC air pump; a glass water trap with a diameter of $2.5 \mathrm{~cm}$ and a useful length of $6 \mathrm{~cm}$; a $0.22 \mu \mathrm{m}$ hydrophobic filter; a $150 \mu \mathrm{m}$ particle filter; a breathing chamber with a volume of $5.0 \times 10-3 \mathrm{~m} 3$ constructed of glass with two tabulations for gas exchange and latex ducts. The Gaslab ${ }^{\circledR}$ software was used to acquire data. In order to evaluate the equipment, Hass avocado fruits (Persea americana) were selected in the degree of physiological maturity with $8.26 \%$ of oil, then conditioned in the breathing chamber with an initial weight of one kilogram, then sealed hermetically to put into operation the equipment to record the respiratory rate during 24 days, at the end of the period, the fruit entered the climacteric process and the commercial maturity was determined with an oil content of $16.06 \%$. The behavior of the respiratory rate of Hass avocado was typical of a climacteric fruit, presenting the climacteric peak on day 20 with 244 x $103 \mathrm{ppm} / \mathrm{Kg}$. H. Physiological weight loss and firmness showed a decreasing behavior during the stage postharvest of the fruits, while the highest concentration of oil, was presented at 24 days postharvest, stage that coincided with the ripeness of consumption

Keywords: Hass avocado; Postharvest; Respiratory rate. 


\section{Introducción}

Los parámetros de maduración con que se recoge la fruta tienen gran influencia sobre el comportamiento durante la poscosecha y la comercialización. Por tanto, el estado de maduración en el que se cosechan los frutos es un factor dependiente de la distancia entre el punto de recolección y el centro de acopio; así, a mayor distancia, los frutos se deben cosechar en un estado de maduración más temprano y viceversa. (Casierra-Posada, García, y Lüdders, 2004).

En esa dirección es sumamente importante la medición de parámetros que influyen en esta etapa tales como la tasa respiratoria, producción de etileno, pérdida de peso, perdida de firmeza, variación de componentes característicos tales como solidos solubles, aceite o cambio de color (López-López y Cajuste-Bontemps, 1999; Osuna García, Beltrán, Vázquez, 2005; Herrera- González, Salazar-García, Gutiérrez-Martínez, y González-Durán, 2013; Balaguera-López, Martínez, Andrea y Herrera-Arévalo, 2014)

Así mismo debido a los requerimientos de calidad de los frutos, es pertinente evaluar su comportamiento en poscosecha, particularmente las características fisiológicas y fisicoquímicas durante la etapa de posrecolección (Márquez, Yepes, Sánchez y Osorio, 2016) de frutos climatéricos como la palta (Olaeta, Undurraga, y Schwartz, 1999). Este fruto está maduro cuando presenta un mínimo de $21,5 \%$ de materia seca en la pulpa y su temperatura óptima de refrigeración es 5,5 으 no mayor a cuatro semanas, a una humedad relativa de 90 a $95 \%$. La tasa de respiración durante la refrigeración es de 10 a $100 \mathrm{~mL} \mathrm{CO} 2 \mathrm{~kg}-1 \mathrm{~h}-1$ y una producción de etileno a $20 \stackrel{\circ}{ } \mathrm{C}>100 \mu \mathrm{L} \mathrm{C2H4} \mathrm{kg-}$ 1h-1 (Dixon et al., 2005; Huysamer y Maré, 2003; Herrera-González, Salazar-García, Martínez- Flores y Ruiz-García, 2017).

Para medir los parámetros la tasa respiratoria expresado en de $\mathrm{CO}_{2}$ se construyó un equipo de medición continua con adquisición de datos a través del software Gaslab.

\section{Objetivo}

El objetivo de la investigación fue evaluar la tasa respiratoria de $\mathrm{CO}_{2}$ a través del sistema experimental de medición, así mimo evaluar los parámetros pos cosecha, como la firmeza, pérdida de peso y contenido de aceite en la palta variedad Hass (Persea americana)

\section{Metodología}

Se construyó un equipo experimental para determinar la intensidad de tasa respiratoria en partes por millón (ppm) de dióxido de carbono ( $\mathrm{CO} 2)$ por unidad de masa y tiempo, para ello se utilizaron: un sensor K-33ICB para medición de CO2; bomba de aire marca Thomas 2000DV/0,5/E/LC para un flujo de aire forzado de 1,5 L/min; una trampa de agua de vidrio de $2,5 \mathrm{~cm}$ de diámetro y largo útil de $6 \mathrm{~cm}$; un filtro hidrofóbico de $0,22 \mu \mathrm{m}$; un filtro de partículas de $150 \mu \mathrm{m}$; una cámara de respiración con un volumen de 5,0 x10-3 m3 construida de vidrio con dos tabuladuras para intercambio gaseoso y conductos de látex, el plano del diseño se puede apreciar en la figura 1. Para el funcionamiento del equipo se verifica el funcionamiento de la bomba y los filtros de partícula y el hidrófobico articulado con la trampa de agua que debe está fijado de forma horizontal en el tablero de control. Para la medición de la tasa respiratoria se pone en funcionamiento el sensor que manda señal al ordenador para registro de datos, paralelamente debe ponerse en funcionamiento la bomba de aire.

Una vez construido el equipo experimental, se seleccionó muestras de paltas Hass, para determinar la cantidad de aceite, otra muestra con peso de $1 \pm 0,5 \mathrm{~kg}$ se lavó con $5 \mathrm{mg}$ de hipoclorito de sodio comercial por litro de agua, luego de secarlos se acondicionó en la cámara de respiración, la que fue sellada de forma hermética para poner en funcionamiento el equipo, el equipo tiene la capacidad de registrar el $\mathrm{CO} 2$ en ppm respecto al tiempo en segundos, la data consistió en registrar 
desde el tiempo de acondicionamiento (madurez fisiológica) hasta el periodo que el fruto entra en climaterio (madurez comercial) por un total de 23 días ( $552 \mathrm{~h}$ ), periodo en el cual el fruto entro en completa senescencia cuando el color del epicarpio vira negro $u$ oscuro.

Además de la medición de tasa respiratoria, se realizaron determinaciones como pérdida fisiológica de peso. La valoración de las características físicas fueron, color externo o de la epidermis e interno o del mesocarpio (Almela et al. 2000). La firmeza se determinó con un penetrómetro para fruta PCE-PTR 200, la medición consistió en la fuerza ejercida durante la penetración de un puntal, en forma de cono, en la pulpa del fruto. Se evaluó cada tres días, en igual cantidad de frutos, las mediciones se hicieron por triplicado.

La pérdida fisiológica de peso se determinó según lo descrito por Barco, Burbano, Mosquera, Villada, Navia (2011), empleando una balanza analítica con desviación de $\pm 0,001 \mathrm{mg}$ por medio de las variaciones a través del tiempo.

Extracción de aceite se realizó según lo descrito por Restrepo-Duque, Londoño-Londoño, González Álvarez, Benavides Paz, Y Cardona Salazar (2012) para el método soxhlet, donde la pulpa se homogeneizó; posteriormente se depositó en cajas de Petri de vidrio, y se extendieron uniformemente en forma de película delgada. Posteriormente, las placas con las muestras se colocaron en una estufa de laboratorio Ecocell 222, a temperatura de $45 \circ \mathrm{C}$ hasta que la pulpa se deshidratara hasta una humedad de $10 \%$ y y luego se llevó a cabo la extracción del aceite utilizando disolvente (hexano) con calor según la norma 963.15 de la AOAC (1990).

\section{Figura 1}

\section{Esquema de equipo de monitoreo post cosecha de frutas y hortalizas}

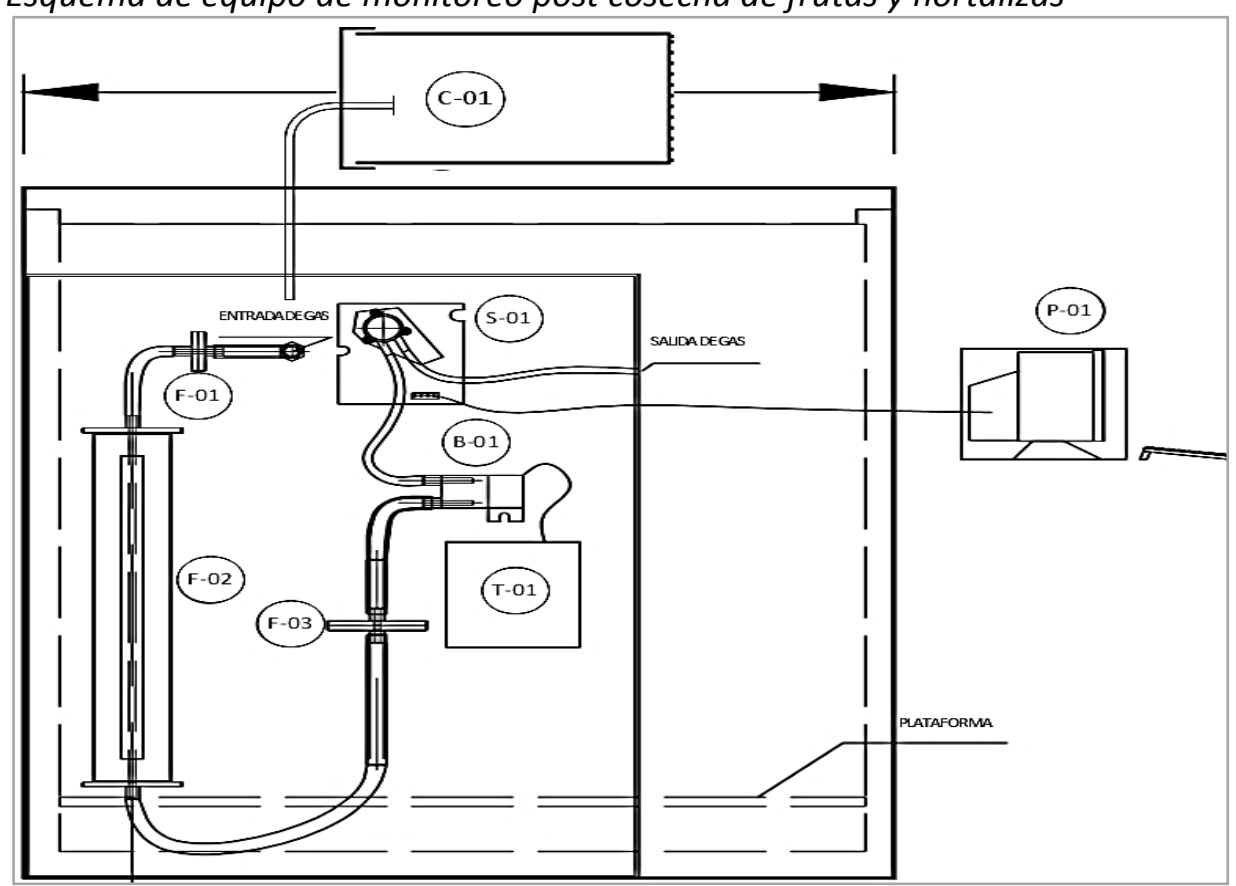

Nota. $\mathrm{C}-01=$ Cámara de vidrio. $\mathrm{P}-01=$ computadora. $\mathrm{S}-01=$ Sensor ICB. B-01 = Bomba. $\mathrm{T}-01=$ Batería 3V. F-03 = Filtro de partículas 2. F-02 $=$ Trampa de agua. F01 = Filtro de partículas 1. Adaptado de "Evaluación de algunos parámetros físicos de palta (Persea americana) en un equipo de medición de postcosecha"

Se graficó la data registrada sobre tasa respiratoria con el software Gaslab, con respecto a los días de medición poscosecha y se analizaron estadísticamente las correlaciones entre alguna variables de interés con el software estadístico Statgraphics centurión XVII. 


\section{Resultados}

La pérdida fisiológica de peso se observa en la figura 2 a, las muestras de palta presentaron un tendencia creciente de pérdida de peso durantetoda el periodo de avaluación, esta tendencia ajustada a una curva se asemeja a una curva polinómica de segundo grado con un coeficiente de regresión de cercano a la unidad $(\mathrm{R} 2=0,99)$, así mismo el análisis de la correlación de Pearson entre las variables resultó altamente significativa $(p=0,02)$. Durante toda la etapa de evaluación postcosecha que comprende desde el acondicionamiento hasta la etapa de senescencia, las muestras tuvieron una pérdida de peso $27,2 \%$, dicho dato se registró en el día 24 cuando finalizó la evaluación.

En la figura $2 \mathrm{~b}$ se puede apreciar la perdida de firmeza de las muestras de palta, a medida que transcurrió el tiempo las muestras presentaron disminución de firmeza. Los valores que corresponden a los primeros días la resistencia a la penetración, la primera medición se efectuó al momento del acondicionamiento de las muestras, siendo el valor $58,3 \mathrm{~N} / \mathrm{m} 2$, una vez acondicionadas en la cámara de respiración, las mediciones sucesivas se efectuaron cada tres días, los valores reportados indican que la firmeza disminuye constantemente y se ajusta a una ecuación polinómica de segundo grado con un coeficiente de regresión cercano a la unidad $(R 2=0,98)$, así mismo un análisis de correlación de Pearson entre la variable firmeza y tiempo resulto significativa $(P=0,00)$, durante la etapa de final de medición se reportó una firmeza de $6,5 \mathrm{~N} / \mathrm{m}^{2}$

\section{Figura 2}

Representación de pérdida fisiológica de peso y variación de firmeza de palta Hass
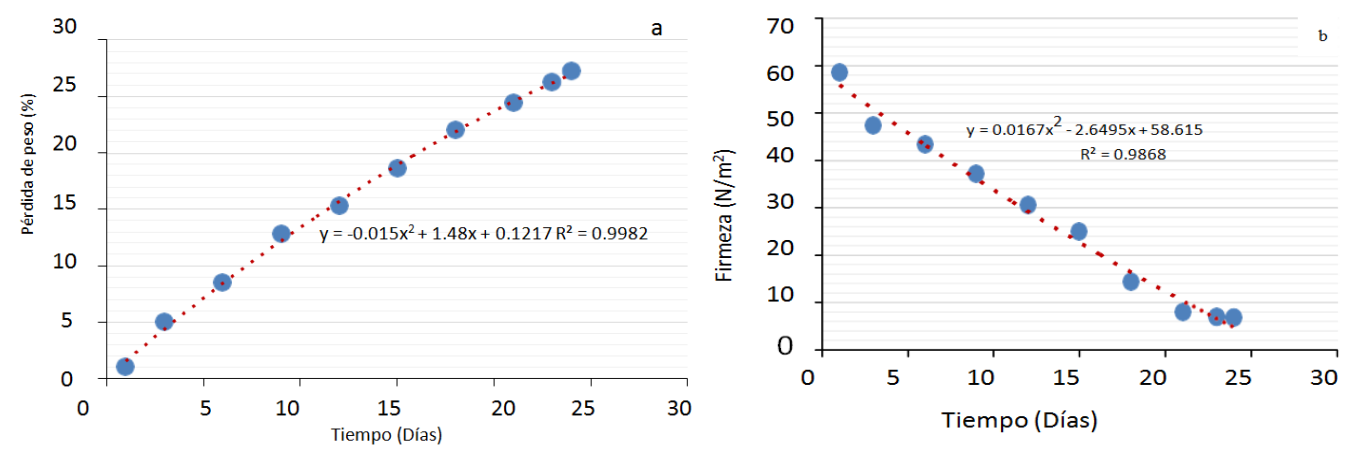

Nota. El gráfico "a" representa la pérdida fisiológica de peso respecto al tiempo de evaluación. El gráfico "b" representa la variación de la firmeza respecto al tiempo de medición. Adaptado de "Evaluación de algunos parámetros físicos de palta (Persea americana) en un equipo de medición de postcosecha"

La cantidad de aceite obtenido en los frutos antes del acondicionamiento en la cámara de respiración fue de $8,26 \%$ en base húmeda, mientras que al finalizar el periodo de evaluación se registró $16,06 \%$, este valor se determinó, considerando que los frutos se presentaban suaves al tacto, indicando su madurez de consumo, este último parámetro se determinó a través de una escala subjetiva que comprendió tres categorías: frutos de textura dura al tacto, frutos cambiantes y frutos suaves, según los recomendado por Zamora, Cajuste, Colina y Santacruz (1999).

La figura 3 presenta la variación de la respiración expresada en ppm de CO2.kg-1.h-1, la tasa de respiratoria de los frutos mostró un primer periodo constante hasta el día 16 y a partir de ese día presentó una aceleración continua y creciente hasta el día 20, pasando de 36 ppm de CO2kg-1h-1 hasta un valor pico de $244 \mathrm{ppm}$ de $\mathrm{CO} 2 \mathrm{~kg}-1 \mathrm{~h}-1$, siendo éste el pico climatérico, posteriormente tuvo un descenso continuo hasta el día 24 cuando presentaron una taza de 48 ppm de CO2 kg-1h-1, posteriormente se dejó de hacer las mediciones por la sobre madurez de las muestras.

En la figura 4, se observan los cambios de aspecto de las muestras de palta con relación al 
epicarpio y mesocarpio, respecto al primero, se observa que en la escala de luminosidad, color y matiz (LCH) reportado por Rossi (2017), una coloración verde azulado $(160,100,50)$ y varia al finalizar a verde oscuro $(160,100,20)$, respecto al mesocarpio se observa que inicialmente cuando se evalúa la madurez fisiológica se encuentran entre el color amarillo $(90,100,75)$ y finalmente cuando presenta madurez comercial vira al ángulo $(50,100,60)$, presentando un color amarillo crema ello ocurre a los 21 días. Posterior a ello puede observarse cambios a colores más oscuros, cuando el fruto ya no presenta cualidades sensoriales a partir de los 23 días.

\section{Figura 3}

Variación de la tasa respiratoria en palta Hass respecto al tiempo

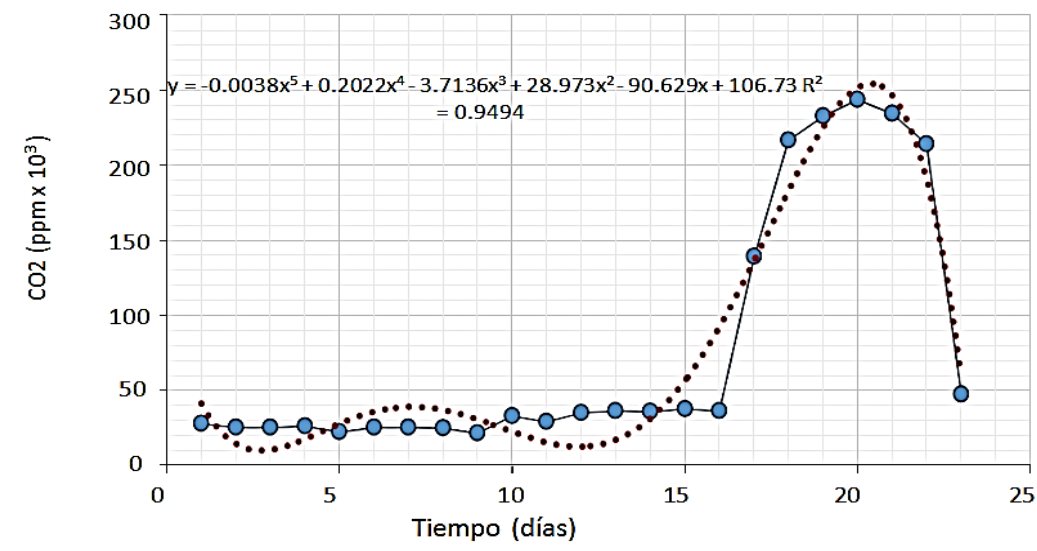

Nota. Las mediciones fueron efectuadas con el equipo de monitoreo de postcosecha construido

\section{Figura 4}

Cambios de aspecto de epicarpio y mesocarpio de paltas (Persea americana) en relación al tiempo evaluado.
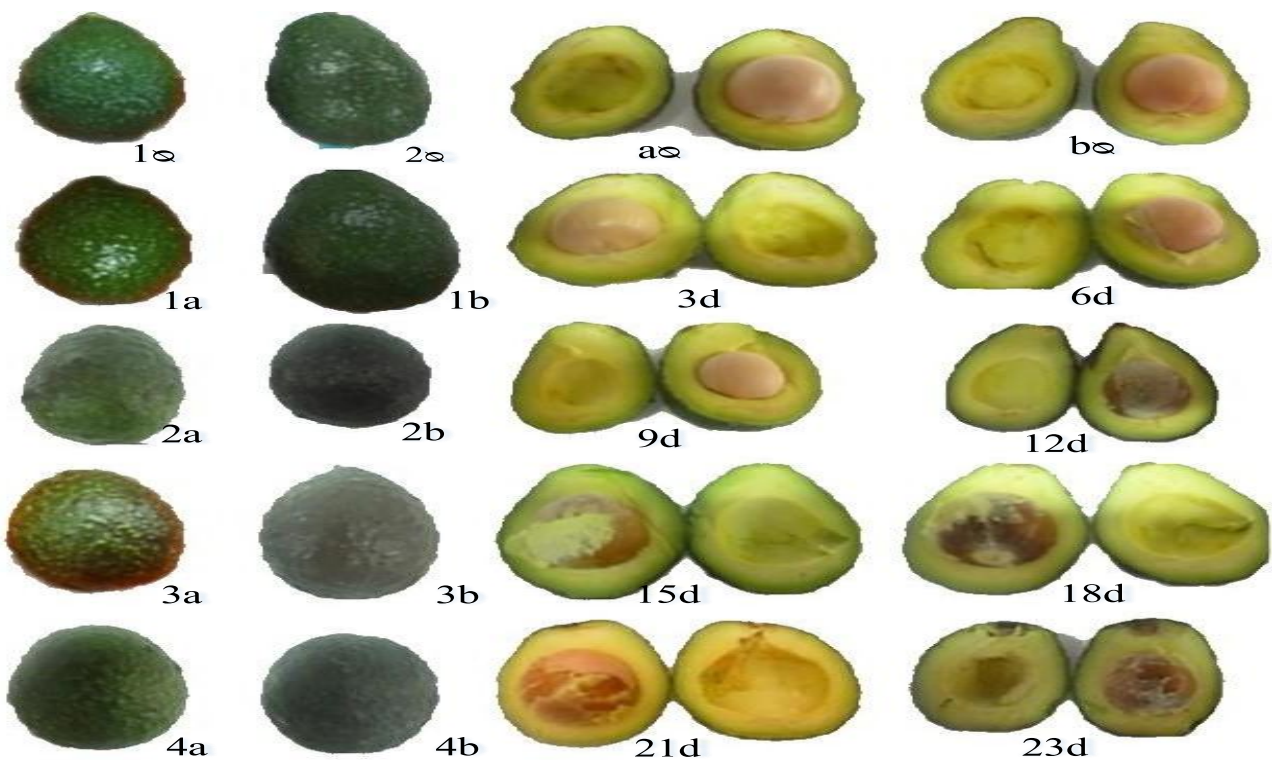

Nota. Las muestras; $1 Q, 2$ y y a y bo representan el aspecto y coloración del epicarpio y mesocarpio respectivamente de los frutos cosechados con madurez fisiológica antes de ser estudiados. Las muestras: 1a, 1b; 2a, 2b; 3a, 3b; 4a, 4b representan la variación del aspecto y coloración durante las cuatro semanas con dos evaluaciones por semana (a y b). Las muestras; 3d, 6d, 9d, 12d, 15d, 18d, 21d, $23 d$ representan la variación de aspecto y color evaluado durante ocho días en cuatro semanas. Adaptado de "Evaluación de algunos parámetros físicos de palta (Persea americana) en un equipo de medición de postcosecha" 


\section{Discusiones}

La pérdida fisiológica de peso registrado durante el tiempo de evaluación, va acompañado de los cambios en la coloración de la piel y pulpa de la fruta, debido al proceso de transpiración, que se ve acrecentado por la disminución de la presión de vapor por efecto de la temperatura de la atmósfera externa que rodea el fruto, la variación de pérdida de peso se presentó directamente proporcional al tiempo de almacenamiento, resultados similares fueron obtenidos en distintas investigaciones (Echeverria y Alonso,1988; Ortega, Villanueva y Sadith, 2011; Villacorta y Vásquez,2016), Respecto a la taza respiratoria, se observó una producción constante de CO2 hasta el día 16, posteriormente se inicia el climaterio (etapa crítica) llegando a un pico máximo de $244 \times 10^{3}$ ppm a los 20 días, luego ocurre un descenso dramático finalizando en $48 \times 10^{3} \mathrm{ppm}$ dichos valores concuerdan con Villa, Molina, Ayala, Guadalupe y González, (2010), quienes encontraron picos similares para paltas Hass, así mismo este comportamiento es calificado como un vegetal perecedero (Kader ,2012) por tener una taza extremadamente alta.

Los picos respiratorios climatéricos son el reflejo de un incremento en la actividad mitocondrial, debido a la mayor disponibilidad de carboxilatos como sustrato, probablemente inducida por el desacomodo ocasionado por el desprendimiento del árbol, combinado con la degradación del almidón, por acción enzimática y la formación de carbohidratos (Márquez, Yepes, Sánchez y Osorio,2016).

Por otro lado, la firmeza de las paltas disminuyeron de manera proporcional a los días transcurridos, desde 58,3 a 28,0 N/m2 siendo similares a los encontrados por Zamora et al (1990).

\section{Conclusiones}

Se construyó un equipo para medir la taza respiratoria de $\mathrm{CO} 2$ que fue empleado en el presente estudio.

El comportamiento de la tasa respiratoria de palta Hass fue típica de un fruto climatérico, presentando el pico climatérico en el día 20 con 244 x 103 ppm/ Kg.h

La pérdida fisiológica de peso y la firmeza, presentaron un comportamiento decreciente durante la etapa postcosecha de los frutos. La mayor concentración de aceite, se presentó a 24 días de postcosecha, etapa que coincidió con la madurez de consumo.

\section{Referencias Bibliográficas}

Márquez, C., Yepes, D., Sánchez, L., \& Osorio, J. (2016). Cambios físico-químicos del aguacate (Persea americana Mill. cv. "Hass") en poscosecha para dos municipios de Antioquia. Temas Agrarios, 19(1), 32-47.

Herrera-González, J., Salazar-García, S., Martínez-Flores, H. y Ruiz-García, J. (2017). Indicadores preliminares de madurez fisiológica y comportamiento postcosecha del fruto de aguacate Méndez. Revista Fitotecnia Mexicana, 40 (3), 325-332.

Dixon J., T. A. Elmsly, D. B. Smith and H. A. Pak (2005). Increasing pick to pack times increases ripe rots in Hass avocados. New Zealand Avocado Growers' Association Annual Research Report5:43-50.

Huysamer M. y L. Maré (2003). The effect of relative humidity and ethyl-ene scrubbing on "Fuerte" and Hass avocado fruit quality. South African Avocado Growers' Association Yearbook26:96-105.

Casierra-Posada, F., García, E. J., y Lüdders, P. (2004). Determinación del punto óptimo de cosecha en el lulo (Solanum. Agronomía Colombiana, 22(1), 32-39.

López-López, L., \& Cajuste-Bontemps, J. F. (1999). Comportamiento postcosecha de fruta de aguacate $\mathrm{cv}$. hass con base en la altitud de producción y tipo de floración. Revista 
Chapingo Serie Horticultura, 5, 365-371.

Osuna García, J., \& Beltrán, J., \& Vázquez Valdivia, V. (2005). Efecto del 1- metilciclopropeno (1MCP) sobre el comportamiento postcosecha del aguacate 'Hass'. Revista Fitotecnia Mexicana, 28 (1), 1-8.

Herrera-González, J. A., Salazar-García, S., Gutiérrez-Martínez, P., y González- Durán, I. J. L. (2013). El comportamiento poscosecha de frutos de aguacate 'Hass' es influenciado por el portainjerto. Revista mexicana de ciencias agrícolas, 4(1), 19-32.

Balaguera-López, H. E., Martínez, C., Andrea, C., y Herrera-Arévalo, A. (2014). Papel del cáliz en el comportamiento poscosecha de frutos de uchuva (Physalis peruviana L.) ecotipo Colombia. Revista Colombiana de Ciencias Hortícolas, 8(2), 181-191.

Olaeta, J., Undurraga, P., \& Schwartz, M. (1999). Determinación de la evolución y caracterización de los aceites en paltas (Persea americana Mill.) cvs. Fuerte y Hass cultivados en Chile. Revista Chapingo Serie Horticultura, 5, 117-122.

Restrepo Duque, A., Londoño-Londoño, J., González Álvarez, D., Benavides Paz, Y Cardona Salazar, B. (2012). Comparación del aceite de aguacate variedad Hass cultivado en Colombia, obtenido por fluidos supercríticos y métodos convencionales: una perspectiva desde la calidad. Revista Lasallista de Investigación, 9 (2), 151161.

AOAC (1990).Association Of Official Analytical Chemists - Aoac. Oficial Methods of analysis. 15thEd. Washington, D. C.

Barco H., P., Burbano D., A., Mosquera S. S., Villada C. H., y Navia P. D. (2011). Efecto del recubrimiento a base de almidón de yuca modificado sobre la maduración del tomate. Revista Lasallista de Investigación, 8 (2), 96103.

Zamora M. T., Cajuste J., Colina M. T., y Santacruz, U. H. (1999). Efecto de los daños mecánicos sobre el comportamiento postcosecha de fruto de aguacate. Rev. Chapingo S. Hort, 5, 319328.

Rossi Tadeo, D. (2017). Estimación de la aceptabilidad visual de la palta (Persea americana Mill.) cv. Hass aplicando estadística de supervivencia.

Villacorta, L. M., \& Vásquez, C. P. (2016). Efecto de las coberturas biodegradables y temperatura sobre el color, firmeza y pérdida de peso en palta (Persea americana Mill) Hass, durante el almacenamiento. Pueblo Continente, 20(2), 379-389.

Ortega Rodríguez, L. J., Villanueva, P., \& Sadith, K. (2011). Efecto del encerado y temperatura de almacenamiento en la conservación postcosecha de la palta (persea americana mill).

Echeverria, D., \& Alonso, R. (1988). Efecto del uso de cera y una pelicula plastica sobre el comportamiento en almacenaje refrigerado de frutos de palta (Persea americana Mill) cv. Fuerte cosechado.

Villa R., Molina C., Ayala Z., Guadalupe I. e González, A. (2010). Effect of maturity stage on the content of fatty acids and antioxidant activity of "Hass" avocado. Food Research International. 44: 1231- 1237.en dos estados de madurez.

Kader, A. (2002). Postharvest technology of horticultural crops. Agriculture and Natural Resources. Davis, California: University of California. 535 p.

\section{Agradecimientos}

Se agradece a la Facultad de Ingeniería de la Universidad Privada de Tacna por el financiamiento de la investigación. 